\title{
A Study of Bone Marrow Aspiration and Biopsy in Adult Patients Presenting with Leukoerythroblastic Blood Picture
}

\author{
Shubha. H. V', Nirmala. $C^{2 *}$ and Dayananda. S. Biligi ${ }^{2}$ \\ ${ }^{1}$ Department of Pathology, Sapthagiri Institute of Medical Sciences and Research Centre, Chikkasandra, Bangalore, Karnataka, India \\ ${ }^{2}$ Department of Pathology, Bangalore Medical College and Research Institute (BMCRI), Bangalore, Karnataka, India
}

\section{ABSTRACT}

Introduction: The presence of leukoerythroblastosisservesas a valuable clue to the presenceof some underlyingserious disease. Various infiltrating \&non-infiltrating conditions present as leukoerythroblasticpicture ranging from infections, sepsis, haemorrhage, haemolysis, anemias to leukemias, lymphomas \&metastases.

Aims and Objectives: To confirm the diagnosis of various conditions presenting with leukoerythroblastosis by studying the bone marrow morphology and to study the distribution of various conditions presenting with leukoerythroblastosisin different age groups and sex.

Materials and Methods: A total of 50 adult patients presenting with leukoerythroblastosiswere studiedduring the period from October 2014 to August2016. The bone marrow aspiration and biopsy were preceded by the evaluation of patient's medical history, clinical and hematological findings withperipheral smear examination for the presence of leukoeythroblastosis. The findings were documentedon a proforma.

Results: 13 (26\%) cases were in the age group of 21-30 years which constituted the largest group. 26 (52\%) were males and 24 (48\%) were females. The spectrum of conditions presenting with leukoerythroblastosis were non-infiltrating conditions withone ( $2 \%)$ normal study, 8 (16\%) erythroid hyperplasias, $21(42 \%)$ megaloblastic anemias, $5(10 \%)$ myelodysplastic syndromes and 1 (2\%) hypoplastic marrow and among the infiltrating conditions were $2(4 \%)$ acute myeloid leukemias, $6(12 \%)$ myeloproliferative disorders, 1 (2\%) metastatic deposit, 2 (4\%) lymphoproliferative disorders and $3(6 \%)$ multiple myelomas.

Conclusion: Leukoerythroblastosis indicates the extent to which bonemarrow reacts to stress and disease. It is characteristic \&diagnostic of a disordered marrow function. Thus, bone marrow aspiration and biopsy help in establishing an early diagnosis and better prognosis of the disease can be expected.

Keywords: Bone Marrow Aspiration, Bone Marrow Biopsy, Leukoerythroblastosis, Myelodysplastic Syndrome, Myelofibrosis

\section{Introduction}

"Leukoerythroblastosis" is defined as the presence of both immature erythroid and myeloid cells in the peripheral blood. ${ }^{[1]}$ Leukoerythroblastic reactions in a general population are frequently caused by nonmalignant disorders such as hemolysis, infection, haemorrhage, megaloblastic anemias, \& combinations of infections, bleeding and hypoxia. ${ }^{[2]}$ Myelophthisis is a form of bone marrow failure due to replacement of haematopoietic tissue by abnormal tissue, most commonly metastatic carcinomas. ${ }^{[3]}$ Myelophthisic anemia can range from an overt leukoerythroblastic picture to the presence of a few tear drop-shaped cells on a blood film. ${ }^{[4]}$

The presence of leukoerythroblastosis serves as a valuable clue to the presence of some underlying disease stressing haematopoiesis or a signal to investigate further for the presence of malignancy. ${ }^{[5]}$ Leukoerythroblastosis in the peripheral blood is a well-recognized manifestation of bone marrow infiltration by malignant tumour.
[6] Although very little is known about the premature release of immature cells in pathologic states, it has been reported that leukoerythroblastosis is more common when fibrosis accompanies tumour in the bone marrow. Myelofibrosis appears to be important in the pathogenesis of leukoerythroblastosis. ${ }^{[7]}$

Leukoerythroblastosis appears to be a nonspecific response of the marrow to a variety of disease processes. It certainly alerts a clinician to the possibility of occult bone metastasis. ${ }^{[8]}$ The present study was conducted to study the spectrum of various conditions presenting with leukoerythroblastosis and to establish the correct diagnosis by studying the bone marrow morphology.

\section{Materials and Methods}

The present study was a prospective study conducted during the period of October 2014 to August 2016 in the department of pathology of Bangalore Medical College and Research Institute, Bangalore. Ethical committee 
clearance was obtained from the institution. A total of 50 patients above the age of 18 years who presented with leukoerythroblastic blood picture in the peripheral smear and with suspected bone marrow pathology based on the haemogram and clinical findings were included in this study. Patients with haemophilia, congenital haemorrhagic disorders or with infections at the potential biopsy site and the patients not giving consent for the bone marrow examination were excluded from the study.

The bone marrow aspiration and biopsy was preceded by the evaluation of medical history, clinical features and hematological findings of the patients. All the findings were documented on a standard proforma. Written and informed consents were taken from the patient/patient's attender after explaining the risks and benefits of the procedure.

\section{Results}

A total of 50 adult patients who presented with leukoerythroblastic picture in the peripheral smear were evaluated. The distribution of the cases presenting with leukoerythroblastic picture according to age (Table 1) \& sex (Table 2) was studied. Out of a total of 50 adult patients maximum number of cases, i.e, $13(26 \%)$ cases were between the age group of 21-30 years. Hence, the patients in this age group constituted the largest group. There were $26(52 \%)$ males and $24(48 \%)$ were females.

Out of 50 cases presenting with leukoerythroblastosis, $1(2 \%)$ was a normal study, $8(16 \%)$ were erythroid hyperplasias, $21(42 \%)$ were megaloblastic anemia (Figure 1), 2 (4\%) were aute myeloid leukemias, 6 (12\%) were myeloproliferative disorders, 5(10\%) were myelodysplastic syndromes (Figure 2 and 3), $1(2 \%)$ was a metastatic deposit (Figure 4), 2 (4\%) were lymphoproliferative disorders, $3(6 \%)$ were multiple myelomas and $1(2 \%)$ was a hypoplastic marrow (Table 3 ).

Most of the cases presenting with leukoerythroblastosis in the peripheral smea had hypercellular marrow (84\%) on bone marrow examination. One of the myelofibrosis cases (fibrotic phase) and both the cases of lymphoproliferative disorder yielded dry tap on aspiration and cellularity was assessed on trephine biopsy.

Table 1: Age specific distribution of the cases presenting with leukoerythroblastosis.

\begin{tabular}{|c|c|c|}
\hline AGE GROUP (in years) & NO. OF CASES & PERCENTAGE \\
\hline $\mathbf{1 8 - 2 0}$ & 04 & 26 \\
\hline $\mathbf{2 1 - 3 0}$ & 13 & 16 \\
\hline $\mathbf{3 1 - 4 0}$ & 08 & 22 \\
\hline $\mathbf{4 1 - 5 0}$ & 11 & 12 \\
\hline $\mathbf{5 1 - 6 0}$ & 06 & 16 \\
\hline $\mathbf{6 1 - 7 0}$ & 08 & 100 \\
\hline TOTAL & 50 & \multicolumn{2}{|c|}{} \\
\hline
\end{tabular}

Table 2: Sex specific distribution of the cases presenting with leukoerythroblastosis.

\begin{tabular}{|c|c|c|}
\hline SEX & NO. OF CASES & PERCENTAGE \\
\hline MALE & 26 & 52 \\
\hline FEMALE & 24 & 48 \\
\hline TOTAL & 50 & 100 \\
\hline
\end{tabular}

Table 3: Spectrum of the haematological disorders presenting with leukoerythroblastosis.

\begin{tabular}{|c|c|c|c|c|}
\hline \multicolumn{3}{|l|}{ DISORDERS } & NO. & PERCENTAGE \\
\hline \multicolumn{3}{|l|}{ 1.Normal study } & 01 & 2 \\
\hline \multicolumn{3}{|l|}{ 2.Erythroid Hyperplasia } & 08 & 16 \\
\hline \multicolumn{3}{|l|}{ 3.Megaloblastic Anemia } & 21 & 42 \\
\hline \multicolumn{3}{|l|}{ 4.AML } & 02 & 4 \\
\hline \multirow{3}{*}{ 5.Myeloproliferative Disorders } & \multicolumn{2}{|c|}{ a) $\mathrm{CML}$} & 02 & 4 \\
\hline & \multirow{2}{*}{ b) MF } & Prefibrotic & 02 & 4 \\
\hline & & Fibrotic & 02 & 4 \\
\hline \multicolumn{3}{|l|}{ 6.Metastatic Deposits } & 01 & 2 \\
\hline
\end{tabular}




\begin{tabular}{|c|c|c|c|}
\hline \multicolumn{2}{|l|}{ DISORDERS } & \multirow{2}{*}{$\frac{\text { NO. }}{02}$} & \multirow{2}{*}{$\frac{\text { PERCENTAGE }}{4}$} \\
\hline 7 Muloduonlostiosundrome & a) $\mathrm{RA}$ & & \\
\hline T.Ivyeloaysplastic synarome & b) RCMD & 03 & 6 \\
\hline \multicolumn{2}{|c|}{ 8.Lymphoproliferative Disorder-NHL } & 02 & 4 \\
\hline \multicolumn{2}{|l|}{ 9.Multiple Myeloma } & 03 & 6 \\
\hline \multicolumn{2}{|l|}{ 10.Hypoplastic marrow } & 01 & 2 \\
\hline \multicolumn{2}{|l|}{ TOTAL } & 50 & 100 \\
\hline
\end{tabular}

Table 4: Comparison of the spectrum of non- infiltrating conditions presenting with leukoerythroblastic blood picture.

\begin{tabular}{|c|c|c|}
\hline DIAGNOSIS & $\begin{array}{c}\text { PRESENT STUDY } \\
\text { (Out of 26 cases) }\end{array}$ & $\begin{array}{c}\text { BURKETT ET AL. } \\
\text { (Out of 42 cases) }\end{array}$ \\
\hline 1. Haemolytic anemia & $02(8 \%)$ & $11(26 \%)$ \\
\hline 2. Infection & $02(8 \%)$ & $08(19 \%)$ \\
\hline 3. Megaloblastic anemia & $21(80 \%)$ & $08(19 \%)$ \\
\hline 4. Haemorrhage & $01(4 \%)$ & $05(12 \%)$ \\
\hline 5. Combined causes & 00 & $10(24 \%)$ \\
\hline
\end{tabular}

Table 5: Comparison of the spectrum of infiltrating conditions presenting with leukoerythroblastic blood picture.

\begin{tabular}{|c|c|c|}
\hline DIAGNOSIS & $\begin{array}{c}\text { OUR STUDY } \\
\text { (Out of 14 cases) }\end{array}$ & $\begin{array}{c}\text { BURKETT ET AL. } \\
\text { (Out of 47 cases) }\end{array}$ \\
\hline 1. Acute leukemia & $02(14 \%)$ & $04(8.5 \%)$ \\
\hline 2. Chronic myeloid leukemia & $02(14 \%)$ & $04(8.5 \%)$ \\
\hline 3. Chronic lymphocytic leukemia & 00 & $05(10.5 \%)$ \\
\hline 4. Other leukemias & 00 & $06(13 \%)$ \\
\hline 5. Lymphoma & $02(14 \%)$ & $08(17 \%)$ \\
\hline 6. Carcinoma & $01(7 \%)$ & $04(8.5 \%)$ \\
\hline 7. Multiple myeloma & $03(22 \%)$ & $06(13 \%)$ \\
\hline
\end{tabular}

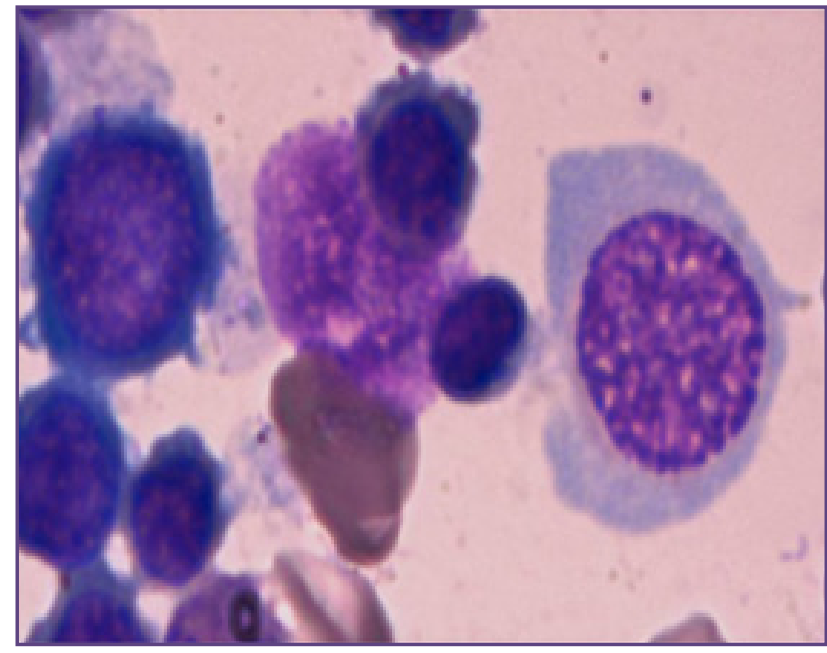

Fig. 1: Bone marrow aspirate showing a Megaloblast with sieve -like chromatin and nuclear cytoplasmic maturation asynchrony. (Leishman's stain; 40X).

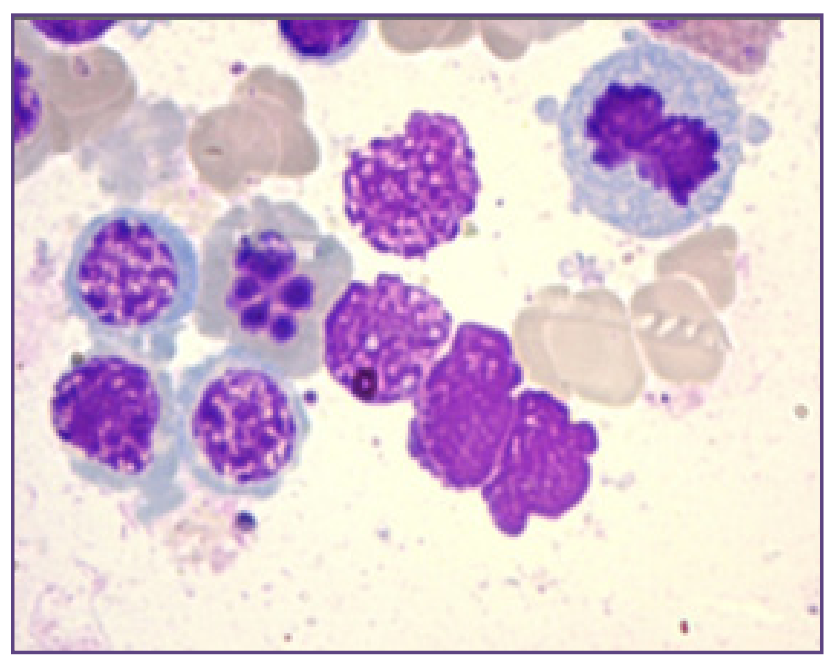

Fig. 2: Bone marrow aspirate in a Myelodysplastic case showing features of dyserythropoiesis such as cytoplasmic blebbing, nuclear budding and abnormal mitotic figure. (Leishman's stain; 40X) 


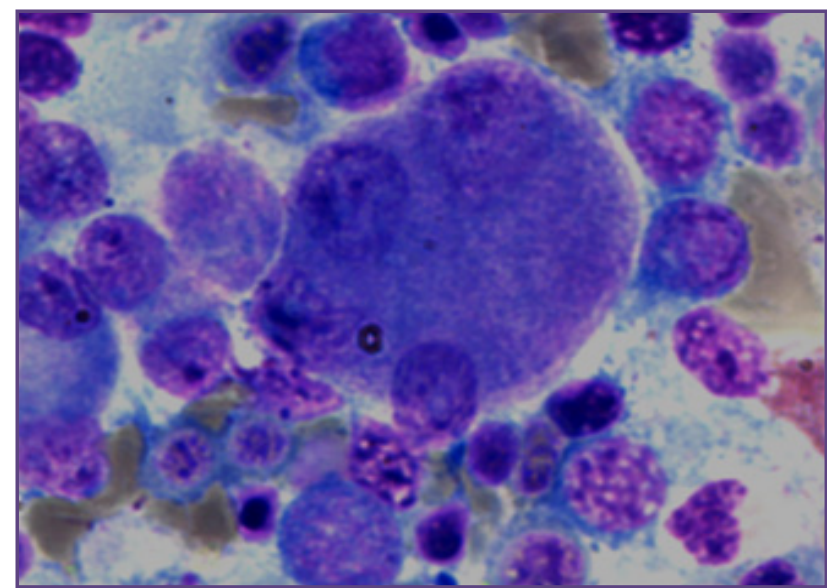

Fig. 3: Bone marrow aspirate showing multiple separated nuclei in a megakaryocyte in a case of Myelodysplastic syndrome. (Leishman's stain; 100X).

\section{Abbreviations}

AML- Acute myeloid leukemia, CML- Chronic myeloid leukemia, MF- Myelofibrosis, RA- Refractory anemia, RCMD- Refractory cytopenia with Multilineage Dysplasia, NHL- Non- Hodgkin's lymphoma).

\section{Discusssion}

The presence of leukoerythroblastic picture serves as a valuable clue to the presence of some underlying disease stressing haematopoiesis or a signal to investigate further for the presence of malignancy. ${ }^{[5]}$ The present study was undertaken to confirm the diagnosis of various conditions presenting with leukoerythroblastosis by studying the bone marrow morphology and to study the distribution of various conditions presenting with leukoerythroblastosis in different age groups and sex.

Bone marrow examination is one of the most valuable diagnostic tests used to evaluate haematological disorders. Bone marrow aspiration and biopsy is useful in establishing the diagnosis when leukoerythroblastic blood picture is present. ${ }^{[9]}$ The spectrum of haematological disorders in patients presenting with leukoerythroblastic blood picture is very wide. Bone marrow examination is, therefore, useful in reaching the final diagnosis.

Leukoerythroblastosis due to infections can mimick leukemia. ${ }^{[10]}$ Bone marrow examination is essential in differentiating these two different entities. A rare cause of leukoerythroblastic anemia was first diagnosed on bone marrow examination as systemic oxalosis. ${ }^{[11]}$ Bone marrow examination is essential to recognize the prefibrotic stages of idiopathic myelofibrosis which presents with leukoerythroblastosis in the peripheral

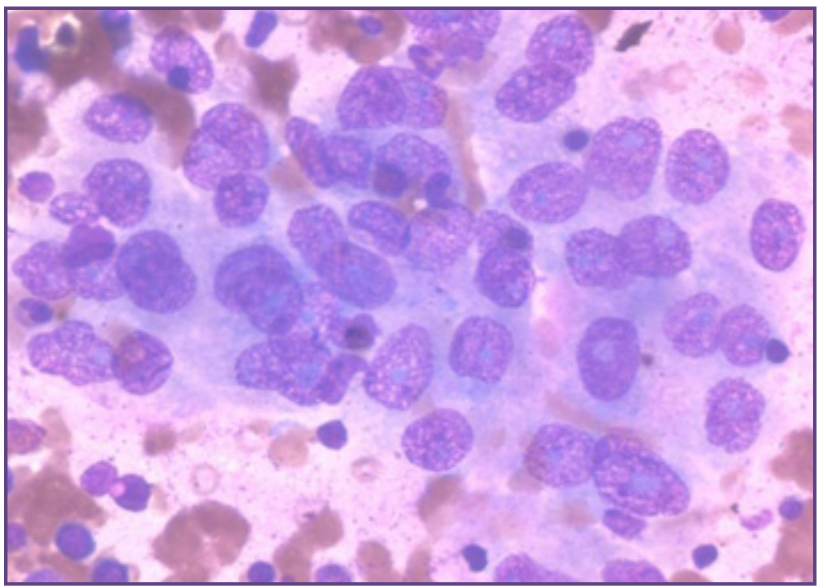

Fig. 4: Bone marrow aspirate showing metastatic deposit consisting of a cluster of large cells with high nuclear cytoplasmic ratio, irregular nuclear borders and prominent nucleoli. (Leishman's stain; 100X).

smear. Biopsy is also essential in establishing the diagnosis of conditions presenting with leukoerythroblastosis which yield a dry tap.

In the present study, out of 50 cases, $13(26 \%)$ cases were between the age group of 21-30 years. Hence, the patients in this age group constituted the largest group who presented with leukoerythroblastic picture in the peripheral smear. 26 $(52 \%)$ were males and $24(48 \%)$ were females. Therefore, no significant differences in the sex distribution of cases was noted. The present study included a wide spectrum of both non-infiltrative and infiltrative conditions. Out of a total of 50 cases:

Non-infiltrative conditions were 26 (52\%) cases which included hemolytic anemias (2), infections (2), megaloblastic anemias (21), haemorrhage (1) (Table 4).

Bone marrow infiltrative disorders were 14 (28\%) cases which included acute myeloid leukemia (2), chronic myeloid leukemia (2), myelofibrosis (4), metastatic carcinoma (1), multiple myeloma (3) and Non-Hodgkin's lymphoma (2) (Table 5).

Burkett et al. evaluated 119 cases of leukoerythroblastosis etiologically. ${ }^{[2]}$ These were divided into the following groups: conditions not associated with marrow infiltration (42 cases) (Table 4), bone marrow infiltrative disorders complicated by another condition which might have produced the leukoerythroblastosis (16 cases) and bone marrow infiltrative disorders (47 cases) (Table 5).

Retief prospectively studied 37 adults with leukoerythroblastosis \& found that one-third were suffering from acute infections, and the rest had various hemolytic states and other non-malignant disorders. ${ }^{[9]}$ In 
a study conducted at the Mayo clinic, out of 37 patients with a leukoerythroblastic picture, $1 / 3^{\text {rd }}$ of the patients had underlying malignancy. ${ }^{[3]}$ Myelophthisis occurs in less than $10 \%$ of cancer patients with metastatic disease. Therefore, the presence of leukoerythroblastic peripheral blood picture serves as a valuable clue to the presence of some underlying disease stressing hematopoiesis as a signal to investigate further for the presence of malignancy. In a case report of multiple bone metastases of carcinoma breast, leukoerythroblastosis was present with intravascular hemolysis. ${ }^{[12]}$

In a study by Thiele et al., in 68 patients in early prefibrotic stage of myelofibrosis, bone marrow histology confirmed a moderate increase in hematopoiesis with a mixed granulocytic and megakaryocytic proliferation, a reduction of erythroid precursors \& significant megakaryocytic abnormalities. These findings were similar to that of our study. ${ }^{[13]}$

In the present study, aspiration was a dry tap in one of the fibrotic phase of myelofibrosis cases and in both the lymphoproliferative disorders. The trephine biopsy was diagnostic in it. In a study done by Humphries, 87 cases of dry tap on marrow aspiration showed significant pathology on trephine biopsies. ${ }^{[14]}$ In a study by Sitalakshmi S et al., 33 out of 176 patients of myelofibrosis revealed dry tap on aspiration. 5 (15\%) of 33 cases were categorised as fibrotic phase of the disease in reticulin stained sections. ${ }^{[15]}$

\section{Conclusion}

The spectrum of haematological disorders in patients presenting with leukoerythroblastic blood picture is very wide. Various malignant \& non-malignant conditions present as leukoerythroblastic picture ranging from infections, sepsis, haemorrhage, haemolysis, anemias to leukemias, lymphomas \& metastases. The presence of leukoerythroblastosis in peripheral smear definitely gives an invaluable clue to the presence of an underlying serious condition and its progression. This necessitates bone marrow examination to determine the cause, evaluate \& analyze the frequency of various conditions presenting with leukoerythroblastosis. A broader background of the patient's clinical and haematological status along with bone marrow examination helps in establishing an early definitive diagnosis and thus, better prognosis of the disease and patient's survival is expected.

\section{Abbreviations}

AML- Acute myeloid leukemia, CML- Chronic myeloid leukemia, MF- Myelofibrosis, RA- Refractory anemia, RCMD- Refractory cytopenia with Multilineage Dysplasia, NHL- Non- Hodgkin's lymphoma.

\section{References}

1. Vaughn JM. Leuco-erythroblastic anaemia. J Pathol Bacteriol 1936;42:541-64.

2. Burkett LL, Cox ML, Fields ML. Leukoeryhtroblastosis in the adult. Am J Clin Pathol 1965;44(5):494-8. DOI: https:// doi.org/10.1093/ajcp/44.5.494

3. Makoni SN, Laber DA. Clinical spectrum of myelophthisis in cancer patients. Am J Haematol 2004;76(1):92-3. DOI: 10.1002/ajh.20046

4. Sook Hee Hong, Myung Woo Lee, Choon Won Kim, Ki Hong Kim. Myelophthisic anemia. Korean J Pathol 1977;11(1):75-83.

5. Clifford GO. The clinical significance of leukoerythroblastic anemia. Med Clin North Am 1966;50(3):779-90. DOI: https://doi.org/10.1016/S0025-7125(16)33177-7

6. Chernow B, Wallner SF. Variables predictive of bone marrow metastases. Cancer 1978;42:2373-78.

7. Rubins JM. The role of myelofibrosis in malignant leukoerythroblastosis. Cancer 1983;51:308-11.

8. Byard RW, Bormains J, Jones TG. Leukoerythroblastosis: A much maligned phenomenon? CMAJ 1987;137(3):191-2.

9. Retief FP. Leuco-erythroblastosis in the adult. Lancet 1964;1:639-42.

10. Aylin CA, Cetin T, Yusuf A, Gulsen K. Leukoerythroblastosis mimicking leukemia: A case report. Iran J Pediatr 2014;24(3):332-3.

11. Naveen K, Divya M, Sheila D, Joseph M, Timothy Rajamanickam. Bone marrow involvement in systemic oxalosis: A rare cause of leukoerythroblastic anemia. Indian J Pathol Microbiol 2011;54(3):659-60. DOI: 10.4103/0377-4929.85145

12. Butler MJ, Yin M, Quddus F. Isolated hemolytic anemia: an unusual manifestation of occult malignancy. Hematol Rep. 2014;6(1):5159. DOI: 10.4081/hr.2014.5159

13. Thiele J, Kvasnicka HM, Zankovich R, Diehl V. Earlystage idiopathic (primary) myelofibrosis- current issues of diagnostic features. Leuk Lymphoma 2002;43(5):1035-41.

14. Humphries JE. Dry tap bone marrow aspiration: clinical significance. Am J Hematol 1990;35(4):247-50.

15. Sitalakshmi S, Anuradha S, Shantala D, Prema D, Betty A. The diagnostic utility of bone marrow trephine biopsies. Indian J Pathol Microbiol 2005;48(2):173-6.

*Corresponding author:

Dr. Nirmala. C, P-101," Vaishnavi Rathnam" Apartment, S.M. Road, Jalahalli cross, Bangalore-560057. India

Phone: +919113576966

Email: drnirmala2018@gmail.com

Financial or other Competing Interests: None. 